\title{
EXTENSIONS OF THE LAPLACE METHOD ${ }^{1}$
}

\section{L. THOMSEN, JR. ${ }^{2}$}

The Laplace method $[1]^{3}$ is concerned with the evaluation of the integral $\int_{0}^{a} \exp [-h \phi(t)] d t(a>0)$ as $h \rightarrow+\infty$. Under suitable conditions on $\phi(t)$ it can be shown that

$$
\int_{0}^{a} \exp [-h \phi(t)] d t \sim \int_{0}^{a} \exp \left[-\frac{1}{2} h \phi^{\prime \prime}(0) t^{2}\right] d t \sim\left(\frac{2 \pi}{h \phi^{\prime \prime}(0)}\right)^{1 / 2} .
$$

We write $A \sim B$ to indicate that $(A-B) / B \rightarrow 0$. In this paper the integral

$$
J=\int_{0}^{a} \exp [-h \phi(t)+k \psi(t)] d t
$$

is considered as $h, k \rightarrow+\infty$ and $a \rightarrow 0+$. Fulks [2] considered the case where $a$ is fixed while $h, k \rightarrow+\infty$ and derived explicit asymptotic expressions assuming suitable conditions on $\phi(t), \psi(t)$ and also assuming that, as $h, k \rightarrow+\infty, k=o(h)$; interesting applications were also given to the incomplete gamma function. Under similar conditions we now derive expressions asymptotic to $J$ as $h, k \rightarrow+\infty$ and in addition as $a \rightarrow 0+$, a case not considered in [2].

It is assumed that the following conditions are satisfied at various parts of the discussion.

(A) $\phi(t)$ is positive and nondecreasing in $0 \leqq t<c . \psi(t)$ is real in $0 \leqq t<c$.

(B) $\phi(t) \in C^{3}, \psi(t) \in C^{2}(0 \leqq t<c)$.

(C) $\phi(t) \in C^{2}, \psi(t) \in C^{2}(0 \leqq t<c)$.

(D) $\phi(t) \in C^{2}, \psi(t) \in C(0 \leqq t<c)$.

(E) $\phi(0)=\phi^{\prime}(0)=\psi(0)=0, \phi^{\prime \prime}(0)>0$.

(F) $k=o(h)$ as $h, k \rightarrow+\infty$.

We propose to investigate how the following integrals approximate $J$ when $h, k \rightarrow+\infty, a \rightarrow 0+$ :

$$
I_{a, h, k}=\int_{0}^{a} \exp \left[-\frac{1}{2} h \phi^{\prime \prime}(0) t^{2}+k \psi^{\prime}(0) t\right] d t,
$$

Received by the editors November 16, 1953.

1 This paper was sponsored in part by the Office of Naval Research, Contract N6onr-244, Task Order XIV at the California Institute of Technology.

2 The author wishes to thank Professor A. Erdélyi of the California Institute of Technology for suggesting this problem and for rendering valuable criticism during the preparation of this paper.

${ }^{3}$ Numbers in brackets refer to references at the end of the paper. 


$$
\begin{aligned}
I_{a, h, k}^{\prime}= & \exp [-h \phi(\tau)+k \psi(\tau)] \\
& \cdot \int_{0}^{a} \exp \left[-\frac{1}{2} h \phi^{\prime \prime}(0)(t-\tau)^{2}\right] d t
\end{aligned}
$$

where $\tau=(k / h)\left(\left(\psi^{\prime}(0) / \phi^{\prime \prime}(0)\right)+O\left(k^{2} / h^{2}\right)\right.$. To obtain $\tau$ one solves the equation $h \phi^{\prime}(\tau)=k \psi^{\prime}(\tau)$ using conditions (B) and (F); see reference [2]. It should be noted here that $I_{a, h, k}, I_{a, h, k}^{\prime}$ may conveniently be expressed as error functions.

We now prove the following theorems:

Theorem 1. If either $\psi^{\prime}(0)<0$ or $k=O\left(h^{1 / 2}\right)$, and conditions (A), (D), (E), (F) are satisfied, then as $h, k \rightarrow+\infty, a \rightarrow 0+$ :

$$
J \sim I_{a, h, k} .
$$

Theorem 2. If $\psi^{\prime}(0)=0$, and conditions (A), (C), (E), (F) are satisfied, then as $h, k \rightarrow+\infty, a \rightarrow 0+$ :

$$
J \sim I_{a, h, k} .
$$

Theorem 3. If $\psi^{\prime}(0)>0, h^{1 / 2}=o(k)$, and conditions (A), (B), (E), (F) are satisfied, then as $h, k \rightarrow+\infty, a \rightarrow 0+$ :

$$
J \sim I_{a, h, k}^{\prime}
$$

provided $(a-\tau) h^{1 / 2}$ is bounded from below.

The statement and proof of Theorem 4 covering the somewhat more complicated general case of $a<\tau$ appear after the proofs of Theorems 1-3.

To prove Theorem 1 we first choose $\epsilon>0$ and for convenience assume $\phi^{\prime \prime}(0)>\epsilon,\left|\psi^{\prime}(0)\right|>\epsilon$. Then we may determine an $\eta$ such that $\left|\phi^{\prime \prime}(0)-\phi^{\prime \prime}(t)\right|<\epsilon,\left|\psi^{\prime}(0)-\psi^{\prime}(t)\right|<\epsilon$ for $0<t<\eta \leqq c$. We then choose $a \leqq \eta$ since $a=o(1)$ by hypothesis. We then have

$\left|\left(J-I_{a, h, k}\right) / I_{a, h, k}\right|$

$=\left|\frac{\int_{0}^{a} \exp [-h \phi(t)+k \psi(t)] d t-\int_{0}^{a} \exp \left[-\frac{1}{2} h \phi^{\prime \prime}(0) t^{2}+k \psi^{\prime}(0) t\right] d t}{I_{a, h, k}}\right|$

$=\left|\frac{\int_{0}^{a}\left\{\exp \left[-\frac{1}{2} h \phi^{\prime \prime}\left(\theta_{1} t\right) t^{2}+k \psi^{\prime}\left(\theta_{2} t\right) t\right]-\exp \left[-\frac{1}{2} h \phi^{\prime \prime}(0) t^{2}+k \psi^{\prime}(0) t\right]\right\} d t}{I_{a, h, k}}\right|$

$\left(0<\theta_{1}, \theta_{2}<1\right)$

$=\left|\frac{\left.\int_{0}^{a} \exp \left[-\frac{1}{2} h \phi^{\prime \prime}(0) t^{2}+k \psi^{\prime}(0) t\right]\right]\left\{\exp \left[-\frac{1}{2} h\left(\phi^{\prime \prime}\left(\theta_{1} t\right)-\phi^{\prime \prime}(0)\right) t^{2}+k\left(\psi^{\prime}\left(\theta_{2} t\right)-\psi^{\prime}(0)\right) t\right]-1\right\} d t}{I_{a, h, k}}\right|$ 
$\leqq\left|\frac{\int_{0}^{a} \exp \left[-\frac{1}{2} h \phi^{\prime \prime}(0) t^{2}+k \psi^{\prime}(0) t\right]\left\{\exp \left[\frac{1}{2} h \epsilon^{2}+k \epsilon t\right]-1\right\} d t}{I_{a, h, k}}\right|$

$\leqq\left|\frac{\epsilon \int_{0}^{a}\left\{\exp \left[-\frac{1}{2} h \phi^{\prime \prime}(0) t^{2}+k \psi^{\prime}(0) t\right] \exp \left[\frac{1}{2} h \epsilon t^{2}+k \epsilon t\right]\right\}\left\{h t^{2}+k t\right\} d t}{\int_{0}^{a} \exp \left[-\frac{1}{2} h \phi^{\prime \prime}(0) t^{2}+k \psi^{\prime}(0) t\right] d t}\right|$

$(4)=\left|\frac{\epsilon \int_{0}^{a h^{1 / 2}} \exp \left[-\frac{1}{2}\left(\phi^{\prime \prime}(0)-\epsilon\right) t^{2}+\frac{k}{h^{1 / 2}}\left(\psi^{\prime}(0)+\epsilon\right) t\right]\left\{t^{2}+\frac{k}{h^{1 / 2}} t\right\} d t}{\int_{0}^{a h^{1 / 2}} \exp \left[-\frac{1}{2} \phi^{\prime \prime}(0) t^{2}+\frac{k}{h^{1 / 2}} \psi^{\prime}(0) t\right] d t}\right|$

or

$(5)=\left|\frac{\epsilon \int_{0}^{a k} \exp \left[-\frac{1}{2} \frac{h}{k^{2}}\left(\phi^{\prime \prime}(0)-\epsilon\right) t^{2}+\left(\psi^{\prime}(0)+\epsilon\right) t\right]\left\{\frac{h}{k^{2}} t^{2}+t\right\} d t}{\int_{0}^{a k} \exp \left[-\frac{1}{2} \frac{h}{k^{2}} \phi^{\prime \prime}(0) t^{2}+\psi^{\prime}(0) t\right] d t}\right|$.

First let us assume $a h^{1 / 2}, a k$ are bounded away from zero (i.e. $a h^{1 / 2}$ $\neq o(1), a k \neq o(1))$. Then we have $\left|\left(J-I_{a, h, k}\right) / I_{a, h, k}\right|=o(1)$ by (4) if $k=O\left(h^{1 / 2}\right)$, and here no restriction is imposed on the sign of $\psi^{\prime}(0)$. If $\psi^{\prime}(0)<0$, the one possibility not taken care of by (4) is when $h^{1 / 2}$ $=o(k)$; in this case we have $\left|\left(J-I_{a, h, k}\right) / I_{a, h, k}\right|=o(1)$ by (5). If we have $a h^{1 / 2}=o(1)$ and/or $a k=o(1)$, (4) and (5) may be rewritten as follows by using the mean value theorem for integrals:

$(4.1)=\left|\frac{\epsilon \exp \left[-\frac{1}{2}\left(\phi^{\prime \prime}(0)-\epsilon\right) t_{1}^{2}+\frac{k}{h^{1 / 2}}\left(\psi^{\prime}(0)+\epsilon\right) t_{1}\right]\left\{t_{1}^{2}+\frac{k}{h^{1 / 2}} t_{1}\right\}}{\exp \left[-\frac{1}{2} \phi^{\prime \prime}(0) t_{2}^{2}+\frac{k}{h^{1 / 2}} \psi^{\prime}(0) t_{2}\right]}\right|$

$$
\left(0<t_{1}, t_{2}<a h^{1 / 2}\right) \text {, }
$$

or

$$
=\left|\frac{\epsilon \exp \left[-\frac{1}{2} \frac{h}{k^{2}}\left(\phi^{\prime \prime}(0)-\epsilon\right) t_{3}^{2}+\left(\psi^{\prime}(0)+\epsilon\right) t_{3}\right]\left\{\frac{h}{k^{2}} t_{3}^{2}+t_{3}\right\}}{\exp \left[-\frac{1}{2} \frac{h}{k^{2}} \phi^{\prime \prime}(0) t_{4}^{2}+\psi^{\prime}(0) t_{4}\right]}\right|
$$

and again we have $\left|\left(J-I_{a, h, k}\right) / I_{a, h, k}\right|=o(1)$ for each of the cases above. This establishes Theorem 1. 
To prove Theorem 2 we proceed in a similar manner. We choose $\epsilon>0$ as before and determine an $\eta$ such that $\left|\phi^{\prime \prime}(0)-\phi^{\prime \prime}(t)\right|<\epsilon$ for $0<t<\eta \leqq c$. We now choose $a \leqq \eta$. We have $\left|\psi^{\prime \prime}(t)\right|<M_{1}$ in $0 \leqq t<c$ since $\psi(t) \in C^{2}$ on $0 \leqq t<c$. Hence we have

$$
\begin{aligned}
\left|\left(J-I_{a, h, k}\right) / I_{a, h, k}\right| & \left|\frac{\int_{0}^{a} \exp [-h \phi(t)+k \psi(t)] d t-\int_{0}^{a} \exp \left[-\frac{1}{2} h \phi^{\prime \prime}(0) t^{2}\right] d t}{I_{a, h, k}}\right| \\
& =\left|\frac{\int_{0}^{a}\left\{\exp \left[-\frac{1}{2} h \phi^{\prime \prime}\left(\theta_{1} t\right) t^{2}+\frac{1}{2} k \psi^{\prime \prime}\left(\theta_{1} t\right) t^{2}\right]-\exp \left[-\frac{1}{2} h \phi^{\prime \prime}(0) t^{2}\right]\right\} d t}{I_{a, h, k}}\right| \\
& \leqq\left|\frac{\int_{0}^{a} \exp \left[-\frac{1}{2} h \phi^{\prime \prime}(0) t^{2}\right]\left\{\exp \left[\frac{1}{2} \epsilon h t^{2}+\frac{1}{2} k M_{1} t^{2}\right]-1\right\} d t}{I_{a, h, k}}\right| \\
& <\left|\frac{\int_{0}^{a} \exp \left[-\frac{1}{2} h \phi^{\prime \prime}(0) t^{2}\right]\left\{\exp \left[\frac{1}{2}\left(\epsilon h+k M_{1}\right) t^{2}\right]\right\}\left(\epsilon h+k M_{1}\right) t^{2} d t}{I_{a, h, k}}\right| \\
& =\left|\frac{\int_{0}^{a h^{1 / 2}} \exp \left[-\frac{1}{2} \phi^{\prime \prime}(0) t^{2}+\frac{1}{2}\left(\epsilon+\frac{k}{h} M_{1}\right) t^{2}\right]\left\{\epsilon+\frac{k}{h} M_{1}\right\} t^{2} d t}{\int_{0}^{a h^{1 / 2}} \exp \left[-\frac{1}{2} \phi^{\prime \prime}(0) t^{2}\right] d t}\right| .
\end{aligned}
$$

The quantity $\left[\phi^{\prime \prime}(0)-\left(\epsilon+(k / h) M_{1}\right)\right]$ can be made positive. Thus we have $J \sim I_{a, h, k}$ unless $a h^{1 / 2}=o(1)$, and this exceptional case may be handled with a mean value theorem for integrals just as in Theorem 1.

In proving Theorem 3 we choose $\epsilon>0$ as in proving Theorem 2 above so that we have $\left|\phi^{\prime \prime}(0)-\phi^{\prime \prime}(t)\right|<\epsilon$ for $0 \leqq t<\eta \leqq c$; we again take $a \leqq \eta$. We have $\left|\psi^{\prime \prime}(t)\right|<M_{1}$ in $0 \leqq t<c$ since $\psi(t) \in C^{2}$ in $0 \leqq t<c$.

$\left|\left(J-I_{a, h, k}^{\prime}\right) / I_{a, h, k}^{\prime}\right|$

$=\left|\frac{\int_{0}^{a} \exp [-h \phi(t)+k \psi(t)] d t-\exp [-h \phi(\tau)+k \psi(\tau)] \int_{0}^{a} \exp \left[-\frac{1}{2} h \phi^{\prime \prime}(0)(t-\tau)^{2}\right] d t}{I^{\prime}{ }_{a, h, k}}\right|$
$=\left|\frac{\int_{0}^{a} \exp \left[\left(-h \phi^{\prime \prime}\left(t_{1}\right)+k \psi^{\prime \prime}\left(t_{1}\right)\right) \frac{(t-\tau)^{2}}{2}\right] d t-\int_{0}^{a} \exp \left[-\frac{1}{2} h \phi^{\prime \prime}(0)(t-\tau)^{2}\right] d t}{\int_{0}^{a} \exp \left[-\frac{1}{2} h \phi^{\prime \prime}(0)(t-\tau)^{2}\right] d t}\right|$

( $t_{1}$ between $t$ and $\tau$ )

$=\left|\frac{\int_{0}^{a} \exp \left[-\frac{1}{2} h \phi^{\prime \prime}(0)(t-\tau)^{2}\right]\left\{\exp \left[-\frac{1}{2} h\left(\phi^{\prime \prime}\left(t_{1}\right)-\phi^{\prime \prime}(0)\right)(t-\tau)^{2}+\frac{1}{2} k \psi^{\prime \prime}\left(t_{1}\right)(t-\tau)^{2}\right]-1\right\} d t}{\int_{0}^{a} \exp \left[-\frac{1}{2} h \phi^{\prime \prime}(0)(t-\tau)^{2}\right] d t}\right|$ 


$$
\begin{aligned}
& \leqq \frac{\int_{0}^{a} \exp \left[-\frac{1}{2} h \phi^{\prime \prime}(0)(t-\tau)^{2}\right]\left\{\exp \left[\frac{1}{2}\left(h \epsilon+k M_{1}\right)(t-\tau)^{2}\right]-1\right\} d t}{\int_{0}^{a} \exp \left[-\frac{1}{2} h \phi^{\prime \prime}(0)(t-\tau)^{2}\right] d t} \mid \\
& =\left|\frac{\int_{-\tau}^{a-\tau} \exp \left[-\frac{1}{2} h \phi^{\prime \prime}(0) u^{2}\right]\left\{\exp \left[\frac{1}{2}\left(h \epsilon+k M_{1}\right) u^{2}\right]-1\right\} d u}{\int_{-\tau}^{a-\tau} \exp \left[-\frac{1}{2} h \phi^{\prime \prime}(0) u^{2}\right] d u}\right| \\
& \leqq\left|\frac{\int_{-\tau h^{1 / 2}}^{(a-\tau) h^{1 / 2}} \exp \left[-\frac{1}{2} \phi^{\prime \prime}(0) t^{2}\right]\left\{\exp \left[\frac{1}{2}\left(\epsilon+\frac{k}{h} M_{1}\right) t^{2}\right]\right\}\left(\epsilon+\frac{k}{h} M_{1}\right) t^{2} d t}{\int_{-\tau h^{1 / 2}}^{(a-\tau) h^{1 / 2}} \exp \left[-\frac{1}{2} \phi^{\prime \prime}(0) t^{2}\right] d t}\right| \\
& (6)=\left|\frac{\left(\epsilon+\frac{k}{h} M_{1}\right) \int_{-\tau h^{1 / 2}}^{(a-\tau) h^{1 / 2}} \exp \left[\frac{1}{2}\left(-\phi^{\prime \prime}(0)+\epsilon+\frac{k}{h} M_{1}\right) t^{2}\right] t^{2} d t}{\int_{-\tau h^{1 / 2}}^{(a-\tau) h^{1 / 2}} \exp \left[-\frac{1}{2} \phi^{\prime \prime}(0) t^{2}\right] d t}\right| \text {. }
\end{aligned}
$$

The quantity $\left[\phi^{\prime \prime}(0)-\left(\epsilon+(k / h) M^{\prime}\right)\right]$ can again be made positive; both integrals in (6) are finite since $1 /\left(\tau h^{1 / 2}\right)=o(1)$, and the upper limit in each integral is bounded from below by hypothesis. Hence we have $J \sim I_{a, h, \mathbf{k}}^{\prime}$. However if $(a-\tau) h^{1 / 2} \rightarrow-\infty,(6)$ is indeterminate, and we must consider another approach as is given below in Theorem 4 .

We consider now in general the case when $a<\tau$; we have $a$ approaching zero faster than $\tau$. The main contribution to $J$ will come from the neighborhood of $t=a$ and not necessarily from the neighborhood of $t=\tau$ as in Theorem 3. Therefore we must consider the expansion of $-h \phi(t)+k \psi(t)$ in powers of $(t-a)$. Also we must have

$$
-h \phi^{\prime}(a)+k \psi^{\prime}(a)>0
$$

since we have a maximum at $t=\tau$ for sufficiently large $h, k$. We adopt the notation:

$$
\begin{aligned}
\beta= & -h \phi^{\prime}(a)+k \psi^{\prime}(a), \\
I_{a, h, k}^{\prime \prime}= & \exp [-h \phi(a)+k \psi(a)] \\
& \cdot \int_{0}^{a} \exp \left\{\left[-h \phi^{\prime}(a)+k \psi^{\prime}(a)\right](t-a)\right. \\
& \left.-\frac{1}{2} h \phi^{\prime \prime}(0)(t-a)^{2}\right\} d t \\
= & \exp [-h \phi(a)+k \psi(a)] \\
& \cdot \int_{0}^{a} \exp \left[\beta(t-a)-\frac{1}{2} h \phi^{\prime \prime}(0)(t-a)^{2}\right] d t .
\end{aligned}
$$


It should be noted that $I_{a, h, \mathrm{t}}^{\prime \prime}$ may also be expressed as an error function. The behavior of $J$ now depends on the quantity $\beta / h^{1 / 2}$, which is more complex than the ratio $k / h^{1 / 2}$ appearing in Theorems $1-3$. We state the following theorem.

TheOREM 4. If $\psi^{\prime}(0)>0, a<\tau$, and conditions (A), (B), (E), (F) are satisfied, then $J \sim I_{a, h, k}^{\prime \prime}$ as $h, k \rightarrow+\infty, a \rightarrow 0+$.

To prove Theorem 4 we choose $\epsilon>0$ so that we have $\left|\phi^{\prime \prime}(0)-\phi^{\prime \prime}(t)\right|$ $<\epsilon$ for $0 \leqq t<\eta \leqq c$; again we have $\left|\psi^{\prime \prime}(t)\right|<M_{1}$ in $0 \leqq t<c$ since $\psi(t) \in C^{2}$ in $0 \leqq t<c$. We have then, on taking $a \leqq \eta$ as before, $\left(J-I_{a, h, k}^{\prime \prime}\right) / I_{a, h, k}^{\prime \prime} \mid$

$=\mid\left\{\exp [-h \phi(a)+k \psi(a)] \int_{0}^{a} \exp \left[\beta(t-a)+\left(-h \phi^{\prime \prime}\left(a_{1}\right)+k \psi^{\prime \prime}\left(a_{1}\right)\right) \frac{(t-a)^{2}}{2}\right] d t\right.$ $\left.-\exp [-h \phi(a)+k \psi(a)] \int_{0}^{a} \exp \left[\beta(t-a)-h \phi^{\prime \prime}(0) \frac{(t-a)^{2}}{2}\right] d t\right\} / I_{a, h, k}^{\prime \prime} \mid$

( $a_{1}$ between $t$ and $a$ )

$=\left|\frac{\int_{0}^{a} \exp \left[\beta(t-a)-h \phi^{\prime \prime}(0) \frac{(t-a)^{2}}{2}\right]\left\{\exp \left(-h\left(\phi^{\prime \prime}\left(a_{1}\right)-\phi^{\prime \prime}(0)\right) \frac{(t-a)^{2}}{2}+k \psi^{\prime \prime}\left(a_{1}\right) \frac{(t-a)^{2}}{2}\right)-1\right\} d t}{\int_{0}^{a} \exp \left[\beta(t-a)-\frac{1}{2} h \phi^{\prime \prime}(0)(t-a)^{2}\right] d t}\right|$

$\leqq\left|\frac{\int_{0}^{a} \exp \left[\beta(t-a)-h \phi^{\prime \prime}(0) \frac{(t-a)^{2}}{2}\right]\left\{\exp \left[\left(\epsilon h+k M_{1}\right) \frac{(t-a)^{2}}{2}\right]-1\right\} d t}{\int_{0}^{a} \exp \left[\beta(t-a)-\frac{1}{2} h \phi^{\prime \prime}(0)(t-a)^{2}\right] d t}\right|$

$=\left|\frac{\int_{0}^{a} \exp \left[-\beta u-h \phi^{\prime \prime}(0) u^{2} / 2\right]\left\{\exp \left[\left(\epsilon h+k M_{1}\right) u^{2} / 2\right]-1\right\} d u}{\int_{0}^{a} \exp \left[-\beta u-h \phi^{\prime \prime}(0) u^{2} / 2\right] d u}\right|$

$\leqq\left|\frac{\int_{0}^{a} \exp \left[-\beta u-h \phi^{\prime \prime}(0) u^{2} / 2\right] \exp \left[\left(\epsilon h+k M_{1}\right) u^{2} / 2\right]\left(\epsilon h+k M_{1}\right) u^{2} d u}{\int_{0}^{a} \exp \left[-\beta u-h \phi^{\prime \prime}(0) u^{2} / 2\right] d u}\right|$

$(8)=\left|\frac{\left(\epsilon+\frac{k}{h} M_{1}\right) \int_{0}^{a h^{1 / 2}} \exp \left[-\frac{\beta}{h^{1 / 2}} u-\frac{1}{2} \phi^{\prime \prime}(0) u^{2}\right] \exp \left[\left(\epsilon+\frac{k}{h} M_{1}\right) \frac{u^{2}}{2}\right] u^{2} d u}{\int_{0}^{a h^{1 / 2}} \exp \left[-\frac{\beta}{h^{1 / 2}} u-\frac{1}{2} \phi^{\prime \prime}(0) u^{2}\right] d u}\right|$

or

$(9)=\left|\frac{\left(\epsilon+\frac{k}{h} M_{1}\right) \int_{0}^{a \beta} \exp \left[-u-\frac{1}{2} \frac{h}{\beta^{2}} \phi^{\prime \prime}(0) u^{2}\right] \exp \left[\left(\epsilon+\frac{k}{h} M_{1}\right) \frac{h}{\beta^{2}} u^{2}\right] \frac{h}{\beta^{2}} u^{2} d u}{\int_{0}^{a \beta} \exp \left[-u-\frac{1}{2} \phi^{\prime \prime}(0) \frac{h}{\beta^{2}} u^{2}\right] d u}\right|$. 
We note that $\epsilon+(k / h) M_{1}=o(1)$ since $\epsilon$ may be chosen arbitrarily small and $k=o(h)$ by hypothesis. Assuming first $a h^{1 / 2} \neq o(1), a \beta \neq o(1)$ we have $J \sim I_{a, h, k}^{\prime \prime}$ by (8) if $\beta=O\left(h^{1 / 2}\right)$ and by (9) if $h^{1 / 2}=o(\beta)$. If $a h^{1 / 2}=o(1)$ and/or $a \beta=o(1)$, we may use a mean value theorem to complete the proof as in Theorem 1 .

It is of interest to evaluate some special cases of the integrals $I_{a, h, k}, I_{a, h, k}^{\prime}, I_{a, h, k}^{\prime \prime}$. For example if $\psi^{\prime}(0)<0$ and $h^{1 / 2}=o(k)$ we have from Theorem 1

$$
J \sim I_{a, h, k} \sim \frac{\left[1-\exp \left(\psi^{\prime}(0) a k\right)\right]}{-k \psi^{\prime}(0)} .
$$

If $a k \rightarrow+\infty,(10)$ would reduce to the result in Theorem 3 of [2]. It will readily be seen that to give more precise results more information must be known about the behavior of $a k$ and $a h^{1 / 2}$, concerning which nothing is assumed in the present discussion. It may be shown from Theorem 3 in this paper that for $a=\tau, \psi^{\prime}(0)>0$, and $h^{1 / 2}=o(k)$,

$$
J \sim I_{a, h, k}^{\prime} \sim \exp [-h \phi(a)+k \psi(a)]\left(\frac{\pi}{2 \phi^{\prime \prime}(0) h}\right)^{1 / 2} .
$$

From Theorem 4 we have for $\psi^{\prime}(0)>0, a<\tau$, and $h^{1 / 2}=o\left(-h \phi^{\prime}(a)\right.$ $\left.+k \psi^{\prime}(a)\right)$,

$$
J \sim I_{a, h, k}^{\prime \prime} \sim \frac{\exp [-h \phi(a)+k \psi(a)]\left\{1-\exp \left[a h \phi^{\prime}(a)-a k \psi^{\prime}(a)\right]\right\}}{-h \phi^{\prime}(a)+k \psi^{\prime}(a)} .
$$

When $a k, a h^{1 / 2} \rightarrow+\infty$, the results will frequently be the same as in [2]; when this is not the case, most of the results explicitly involving $a$ will be expressible as error functions with the exception of a few similar to those above.

\section{REFERENCES}

1. D. V. Widder, The Laplace transform, Princeton, 1946.

2. W. Fulks, A generalization of Laplace's method, Proc. Amer. Math. Soc. vol. 2 (1951) pp. 613-622.

California Institute of Technology and

The Pennsylvania State University 